\title{
Orobanche flava (Orobanchaceae) in Poland: current distribution, taxonomy, hosts and plant communities
}

\author{
Renata Piwowarczyk
}

Department of Botany, Institute of Biology, Jan Kochanowski University, Świętokrzyska 15, 25-406 Kielce, Poland, e-mail: renata.piwowarczyk (a)ujk.edu.pl

\begin{abstract}
Orobanche flava is a species of Central European mountain ranges, mainly the Alps and Carpathian Mts. The paper presents the current distribution of $O$. flava in Poland based on a critical revision of herbarium and literature data as well as results of field investigations conducted between 1999 and 2014. The distribution of species is centered in southern Poland, mainly in the Carpathian Mts., and, sporadically, in the Sudeten Mts. The distribution of O. flava in Poland is mapped. The taxonomy, biology, and ecology are also discussed.
\end{abstract}

Key words: Orobanche flava, Orobanchaceae, taxonomy, distribution, phytocoenoses

\section{Introduction}

The holoparasitic genus Orobanche L. s.l. (Orobanchaceae) in Poland contains 19 species, including two ephemerophytes (Halamski 2005; Piwowarczyk 2006, 2010, 2011, 2012a, 2012b, 2012c, 2012d, 2012e, 2012f, 2012g, 2012h, 2013; Piwowarczyk \& Kirpluk 2011; Piwowarczyk \& Przemyski 2009, 2010; Piwowarczyk et al. 2009, 2010, 2011). O. flava Mart. ex F. W. Schultz, butterbur broomrape, is a Central European montane species, mainly Alpine-Carpathian. It occurs in the mountains of Central Europe from the Pyrenees, the Alps, the Sudeten and Carpathian Mts., and, most probably, in the Atlas in North West Africa (O. flava var. doriae) and in the Caucasus (Meusel et al. 1978; Pusch \& Günther 2009).

In Poland, it grows in the Carpathian Mts. and, sporadically, in the Sudeten Mts. (Tyszkowski 1993; Zázvorka 1999; Zając \& Zając 2001). It has been very rarely reported from lowland areas (Dajdok et al. 1998; Wayda 2001; Zając \& Zając 2001; Michalewska 2004) and anthropogenic sites, i.e. the Botanical Garden in Warsaw (Piwowarczyk \& Kirpluk 2011). It is reported as a parasite of the Asteraceae family species, chiefly Petasites kablikianus, P. paradoxus, P. hybridus, P. albus, Tussilago farfara, Adenostyles sp., Senecio doria (O. flava var. doriae) (Kreutz 1995; Uhlich et al. 1995).
The aim of this study was to identify the current distribution of Orobanche flava in Poland based on field investigations and verified herbarium and literature data. Preferred habitats, communities, hosts, taxonomic problems and threats to the species were also discussed below.

\section{Taxonomic remarks}

Orobanche flava Mart. ex F. W. Schultz Beitr. Kenntn. Deutsch. Orob. 9, 1829. - Syn. Orobanche tussilaginis Mutel, Fl. Franç. 2: 349, 1835; Mutel, Atlas, p. 42, fig. 310, 1837; O. fröhlichii Rchb. fil., Icon. fl. Germ. 20: 106, p. 207, 1862; O. petasites Borbás in Österr. Bot. Zeitschr. 35: 76, 1885; O. petasitis A. J. C. v. Fischer, Mitt. Naturf. Ges. Bern 87/88: 6, 1847.

Type: Germany, Bavaria, "in Gebüschen, feuchten schattigen Hainen bei München auf den Wurzeln von Berberis vulgaris und wahrscheinlich auch anderer Sträucher" (Schultz 1829, p. 9). First collected there by Ritter v. Martius in 1818 ("Monachii prope Ys. Hesselloh").

Although several varieties and forms of Orobanche flava have been described, they require verification. They include the endemic O. flava var. doriae Emberger \& Maire from Morocco (Great Atlas) parasitic on Senecio doria (Emberger 1935-1936). Also based on 
the length of the corolla tube, further forms are distinguished: one with large flowers, up to $20 \mathrm{~mm}$ long, O. flava f. petasitidis Beck and f. communis Beck; and one with smaller flowers, 15-17 mm, f. adenostylidis Beck, additionally reported on Adenostyles from Romania and Austria (Beck 1922, 1930).

An interesting subspecies from the Caucasus (Adygaea), probably endemic and parasitic on Cicerbita petiolata, described as Orobanche flava subsp. cicerbitae Uhlich \& Rätzel (Rätzel \& Uhlich 2004) also requires further observations.

The studied species is rather homogenous within its entire range. However, it occurs very often in different colour forms, even within one population. The typical form, ochre, yellowish, light brown, is the most frequent one, but light-yellow, pink, raspberry or light-red forms are also recorded. Several colour forms have been described: Orobanche flava f. versicolor G. Grintescu - flowers and bracts in lower spike violet-yellowish, on P. hybridus; f. persicina G. Grintescu - flowers and entire shoots peach-coloured, on P. paradoxus; f. flavorosea G. Grintescu - flowers yellow-pink, stem yellow, on P. albus (Grintescu 1945a, 1945b).

Due to similar habitats they colonize, Orobanche flava can also be mistaken with other species. These are: $O$. lycoctoni Rhiner, a montane species parasitic on Aconitum lycoctonum with a disjunctive range comprising mostly Cantabria (Spain) and the Alps, and O. salviae F.W. Schultz parasitic on Salvia glutinosa, chiefly in the Alps and south-western Carpathian Mts. Unless information regarding the host or the habitat is included, $O$. flava in the herbarium material can be easily mistaken with other species of grex Curvatae (Beck 1890), especially O. alsatica, O. bartlingii, O. elatior $\mathrm{s}$. str. or O. lucorum (e.g. Zázvorka 1997; Piwowarczyk 2012c).

\section{Material and methods}

Field investigations were conducted in 1999-2014. They were intensified in 2006-2013. Simultaneously, I revised herbarium materials of Orobanche flava collected from Poland. The materials are deposited in Polish herbaria CHRZ, KRA, KRAM, KTC, KTU, LBL, LOD, OPOL, POZ, TRN, WA, but also in Germany (B, GLM), Switzerland (G), Austria (W), Russia (LE) and in private herbaria.

Herbarium acronyms are given after Mirek et al. (1997) and Holmgren and Holmgren (1998). The nomenclature of vascular plants follows Mirek et al. (2002). The nomenclature of syntaxa is based on Matuszkiewicz (2006). Localities are listed alphabetically as ATPOL cartogram units $(10 \mathrm{~km} \times 10 \mathrm{~km}$, based on Zając 1978; see http://www.ib.uj.edu.pl/chronpol/). They are described as follows: ATPOL grid unit, geographic location, habitat description, abundance in brackets. The following information is also given for most localities: geographic coordinates and altitude (above sea level), and, for revised exsiccata, the collector and collection date, exsiccatum number and the herbarium acronym.

Host plants were also observed by delicately exposing the soil with a gardening shovel. A total of 50 localities were observed in the field (also at sites in Slovakia: Nízké Tatra Mts., Malá Fatra and Velká Fatra mountain ranges; and Ukraine: in the East Carpathian Mts. - in Czarnohora near Bystrec village) and in the analysis of herbarium materials containing an attached host.

\section{Results}

\subsection{Distribution in Poland}

In Poland, Orobanche flava is a species of the montane belt occurring mostly in the Carpathian Mts. and, sporadically, in the Sudeten Mts. It has been very rarely reported from lowland areas (Dajdok et al. 1998; Wayda 2001; Zając \& Zając 2001; Michalewska 2004). The locality in the Botanical Garden in Warsaw is outside the species' Carpathian native range and is one of the northernmost sites in Poland (Piwowarczyk \& Kirpluk 2011), (Fig. 1).

Verified data collected from herbarium materials, literature and unpublished materials are given on the list of localities (Appendix 1). Unlike other species of the Orobanche genus in Poland, O. flava is a taxon difficult to mistake due to its specific occurrence area, chiefly associated with the montane regions, habitats and hosts. The verified distribution of the species in Poland presented in my study was slightly modified in comparison with the distribution map given by Zając and Zając (2001).

\subsection{Preferred habitats and plant communities}

Orobanche flava most frequently grows in tall herbs near streams in Petasites scrubs on wet, stony floodplains usually located above the water level, sporadically flooded, chiefly on fertile soils, rich in calcium carbonate. It is found less frequently in nitrophilous or weakly acidic communities of perennial plants and on margins of wet forest communities; also in synanthropic habitats, i.e. tall herb roadsides, alluvia, on rock debris, Carpathian alder forest. In the Carpathian Mts., it is characteristic of the montane-belt Agropyro caninae-Petasitetum kablikiani association (syn. Petasitetum kablikiani) of the Betulo-Adenostyletea class (Matuszkiewicz 2006), in which it reaches the occurrence optimum. It is found less often in scrub with dominant Petasitetum albi (class Betulo-Adenostyletea) and in the Phalarido-Petasitetum hybridi association of 


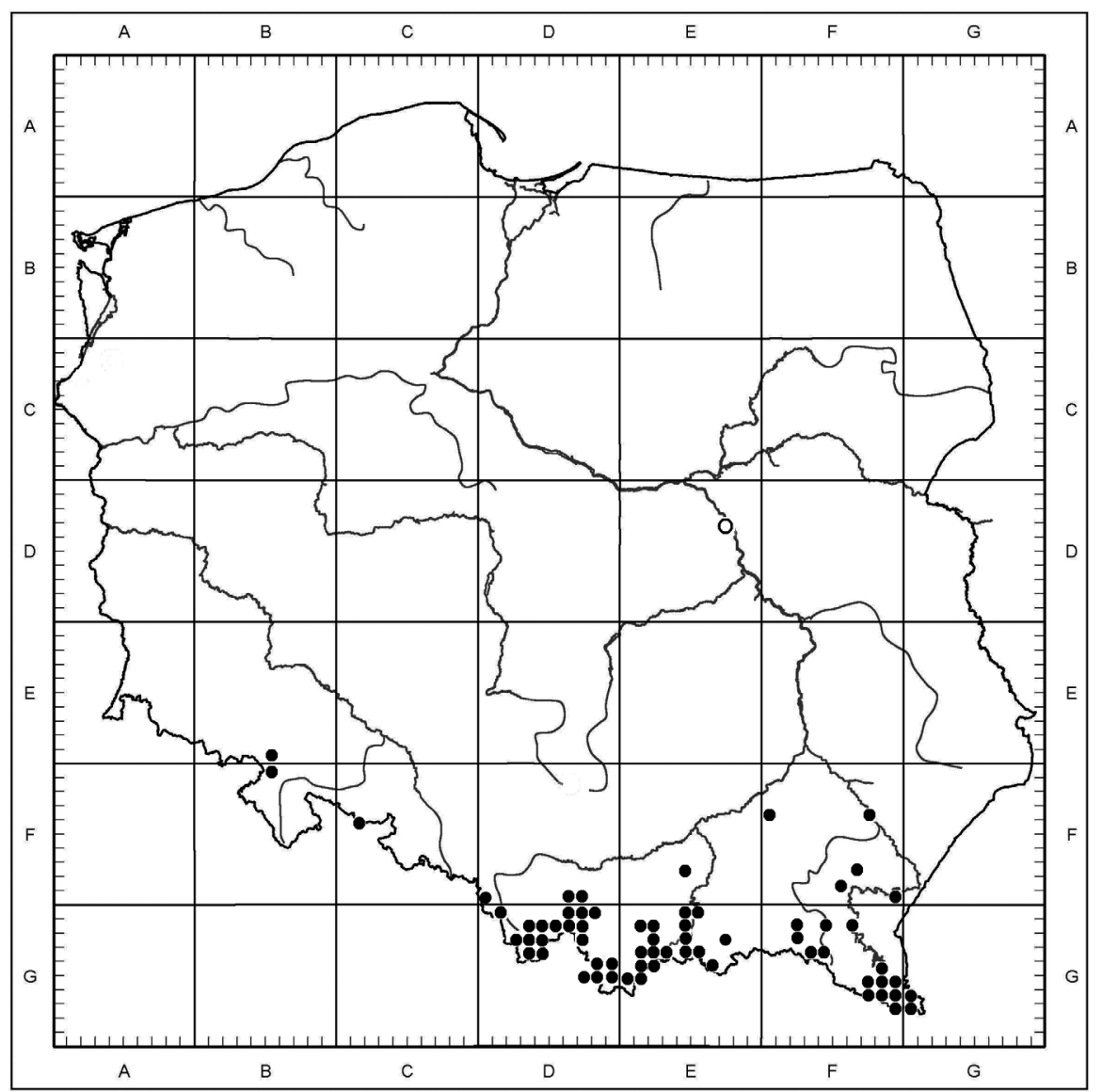

Fig. 1. Distribution of Orobanche flava in Poland

Explanations: • - native locality, ○ - synanthropic locality, probably unknowingly introduced

the Artemisietea vulgaris class. In the Sudeten Mts., it grows on the Biała stream in the Opawskie Mts. in the Phalarido-Petasitetum hybridi association (Tyszkowski 1993). It is often recorded in ecotones of the Carpathian alder forest Alnetum incanae, riparian willow forest Salicetum albo-fragilis, with a contribution of species of the Querco-Fagetea class (especially in lower layers), Vaccinio-Piceetalia (class Vaccinio-Piceetea), and also Molinio-Arrhenatheretea, with a contribution of ruderal species of the Artemisietea vulgaris class (Table 1). In the lowland, in the Lower San valley, it was also recorded in wet tall herbs with a contribution of Petasites hybridus (Michalewska 2004). It grows in a patch of $P$. hybridus in the Botanical Garden in Warsaw (Piwowarczyk \& Kirpluk 2011). It occurs on a variety of soils, on metamorphic shale, dolomites, limestone, Carpathian flysch, rarely basalts. The altitude optimum is recorded less frequently in the foothill belt and most frequently within the lower montane belt, 360-1005 m.

\section{Discussion and conclusions}

In Poland, Orobanche flava grows in the Carpathian Mts. and, sporadically, in the Sudeten Mts. It is a species of the montane belt. O. flava was recorded in lowland areas very rarely (Dajdok et al. 1998; Wayda 2001; Zając \& Zając 2001; Michalewska 2004). The species was introduced into the Botanical Garden in Warsaw; it lies far outside the native range of the species and is the northernmost site of its occurrence in Poland (Piwowarczyk \& Kirpluk 2010). O. flava was reported from botanical gardens in, e.g., Dresden, Berlin or Oxford, where it often co-occurred with $O$. lucorum (Rumsey \& Jury 1991; Pusch \& Günther 2009;). It is also cultivated in the Mountain Botanical Garden in Zakopane in Poland (Nowak et al. 2000). To date, it has not spread outside botanical gardens.

Orobanche flava is a montane taxon with a characteristic and narrow range, habitat and preferred hosts. Unlike other species of the Orobanche genus, its verified distribution changed only slightly as compared to the distribution map given in a study by Zając and Zając (2001). Other species of the Orobanche genus also occurred in the montane area in Poland, especially in the Carpathian Mts., i.e. O. mayeri, O. caryophyllacea, O. elatior, O. minor, O. alba, O. reticulata (incl. O. pallidiflora), sporadically, O. lutea (e.g. Bartoszek \& Piwowarczyk 2008; Nejfeld \& Bartoszek 2008; Piwowarczyk et al. 2010; Piwowarczyk 2011, 2012a, 2012b, 2012c, 2014; Piwowarczyk \& Kirpluk 2011). In 
Table 1. Plant communities with Orobanche flava in Poland

\begin{tabular}{|lccccccccccccccccccccc}
\hline No. of relevé & 1 & 2 & 3 & 4 & 5 & 6 & 7 & 8 & 9 & 10 & 11 & 12 & 13 & 14 & 15 & 16 & 17 & 18 & 19 & $\mathrm{C}$ \\
\hline Area of relevé $\left(\mathrm{m}^{2}\right)$ & 25 & 10 & 20 & 25 & 25 & 25 & 30 & 30 & 40 & 30 & 30 & 30 & 50 & 50 & 40 & 50 & 40 & 50 & 50 & $\mathrm{n}$ \\
Cover of tree layer A (\%) & 70 & 20 & 20 & 20 & 30 & 60 & 20 & 15 & 25 & - & - & 30 & - & - & 30 & 20 & 20 & 40 & 45 & $\mathrm{~s}$ \\
Cover of shrub layer B (\%) & 15 & - & 15 & - & 15 & 15 & 10 & - & 10 & - & 10 & 15 & - & 10 & 20 & 25 & 15 & 15 & 15 & $\mathrm{t}$ \\
Cover of herbaceus layer C (\%) & 65 & 75 & 75 & 100 & 95 & 90 & 85 & 90 & 90 & 85 & 90 & 95 & 100 & 90 & 95 & 85 & 85 & 95 & 90 & $\mathrm{a}$ \\
Cover of moss layer D (\%) & 5 & 15 & 15 & 15 & 10 & 15 & 20 & 30 & 15 & 15 & 15 & 15 & 30 & 15 & 15 & 15 & 30 & 30 & 25 & $\mathrm{n}$ \\
Number of species & 25 & 8 & 17 & 15 & 20 & 24 & 26 & 25 & 21 & 19 & 18 & 18 & 35 & 33 & 27 & 28 & 29 & 33 & 35 & $\mathrm{y}$ \\
\hline
\end{tabular}

ChAss. Petasitetum kablikiani

Orobanche flava

Petasites kablikianus

$\begin{array}{rlllllllllllllllllllllllll}+ & + & + & + & + & + & + & + & + & + & \mathbf{1} & + & \mathbf{1} & \mathbf{1} & + & + & + & + & + & \mathbf{V} \\ . & . & 4 & 4 & 5 & 4 & 3 & 4 & 5 & 5 & 5 & 5 & 5 & 4 & 4 & 4 & 3 & 4 & 4 & V\end{array}$

Ch. Adenostylion alliariae, Betulo-Adenostyletea

Chaerophyllum hirsutum

Senecio nemorensis s.1.

Aconitum firmum cfr.

Phyteuma spicatum

Senecio subalpinus

Carduus personata

Cicerbita alpina

Hypericum maculatum

Petasites albus

Calamagrostis arundinacea

Thalictrum aquilegiifolium

Ch. Fagetalia, Querco-Fagetea

Primula elatior

Astrantia major

Lysimachia nemorum

Acer pseudoplatanus A

Acer pseudoplatanus $\mathrm{C}$

Carex sylvatica

Hordelymus europaeus

Impatiens noli-tangere

Acer pseudoplatanus B

Alnus incana A

Alnus incana $\mathrm{C}$

Fagus sylvatica B

Geranium phaeum

Stachys sylvatica

Asarum europaeum

Caltha laeta

Cirsium erisithales

Corylus avellana B

Fagus sylvatica $\mathrm{C}$

Galeobdolon luteum

Salvia glutinosa

Sanicula europaea

Stellaria nemorum

Ch. Molinio-Arrhenatheretea

Prunella vulgaris

Dactylis glomerata

Taraxacum officinale agg.

Ranunculus repens

Trifolium pratense

Cirsium oleraceum

Achillea millefolium

Leontodon autumnalis

Rumex acetosa

Trifolium repens

Bellis perennis

Myosotis palustris

Ranunculus acris

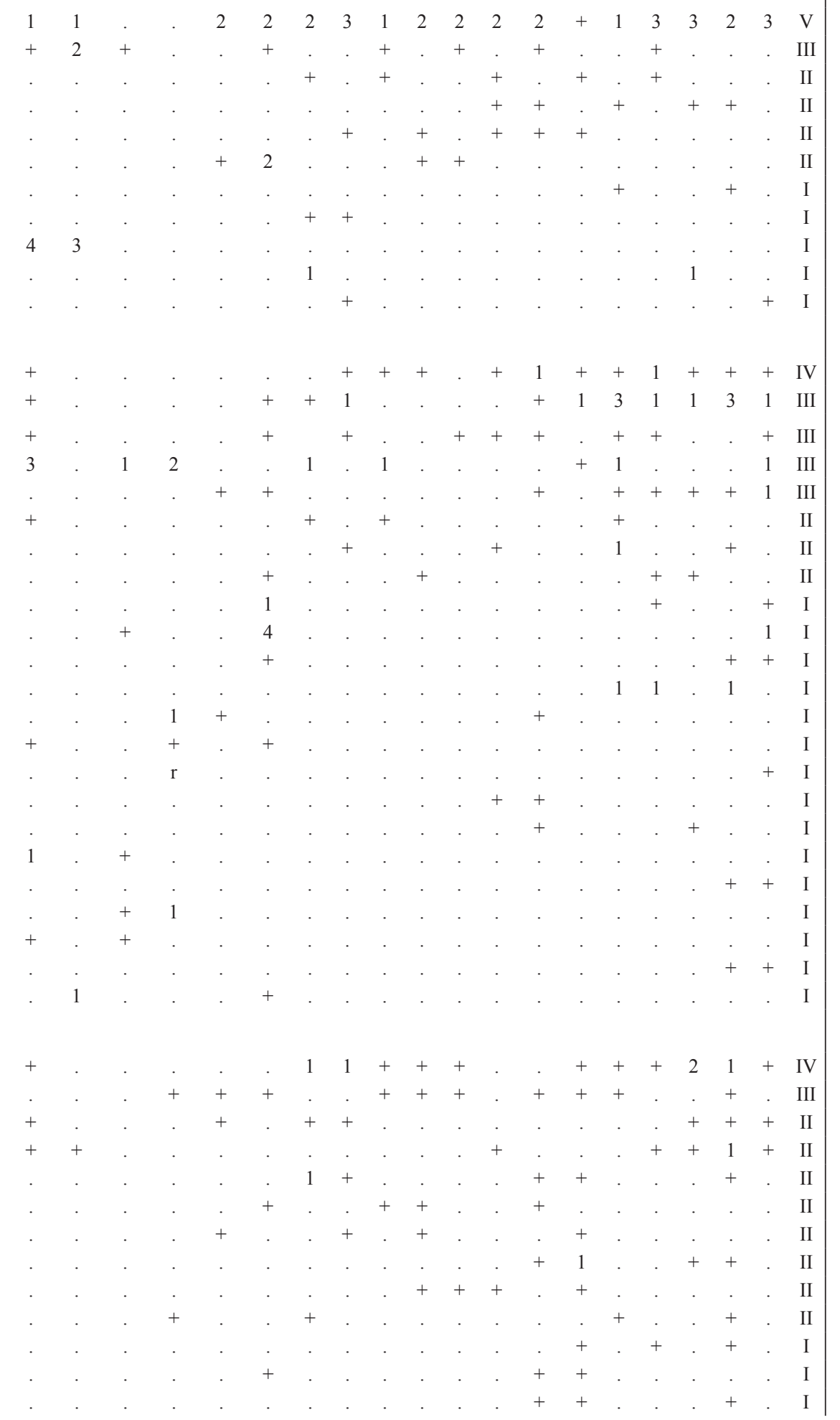




\begin{tabular}{|c|c|c|c|c|c|c|c|c|c|c|c|c|c|c|c|c|c|c|c|c|}
\hline No. of relevé & 1 & 2 & 3 & 4 & 5 & 6 & 7 & 8 & 9 & 10 & 11 & 12 & 13 & 14 & 15 & 16 & 17 & 18 & 19 & $\mathrm{C}$ \\
\hline Cover of tree layer A (\%) & 70 & 20 & 20 & 20 & 30 & 60 & 20 & 15 & 25 & - & - & 30 & - & - & 30 & 20 & 20 & 40 & 45 & $\mathrm{~s}$ \\
\hline Cover of herbaceus layer C $(\%)$ & 65 & 75 & 75 & 100 & 95 & 90 & 85 & 90 & 90 & 85 & 90 & 95 & 100 & 90 & 95 & 85 & 85 & 95 & 90 & $\mathrm{a}$ \\
\hline Cover of moss layer D (\%) & 5 & 15 & 15 & 15 & 10 & 15 & 20 & 30 & 15 & 15 & 15 & 15 & 30 & 15 & 15 & 15 & 30 & 30 & 25 & II \\
\hline Cirsium rivulare & . & . & . & . & . & . & . & . & . & . & . & . & + & + & . & . & . & . & . & $\mathrm{I}$ \\
\hline Euphrasia rostkoviana & . & . & . & . & . & . & . & . & . & + & . & . & . & + & . & . & . & . & . & I \\
\hline Polygonum bistorta & . & . & . & . & . & . & . & . & . & . & . & . & + & + & . & . & . & . & . & I \\
\hline Filipendula ulmaria & . & . & . & . & . & + & . & . & . & . & . & . & + & . & . & . & . & . & . & I \\
\hline Lysimachia vulgaris & . & . & . & + & . & . & . & . & . & . & . & . & + & . & . & . & . & . & . & I \\
\hline Luzula sylvatica & . & . & . & . & . & . & + & . & + & . & . & + & + & . & + & + & . & + & + & III \\
\hline Homogyne alpina & . & . & . & . & . & . & . & . & . & + & + & . & + & . & + & + & + & . & + & II \\
\hline Picea abies $\mathrm{C}$ & . & . & . & . & . & . & . & . & + & . & . & + & + & . & + & . & + & + & + & II \\
\hline Picea abies B & . & . & . & . & . & . & + & . & 1 & . & . & 1 & . & . & 2 & . & 1 & . & 1 & II \\
\hline Leucanthemum waldsteinii & . & . & . & . & . & . & . & + & . & . & . & . & 1 & 1 & + & 1 & + & . & . & II \\
\hline Abies alba $\mathrm{C}$ & + & . & . & . & . & . & . & . & . & . & . & . & . & . & + & . & . & + & + & II \\
\hline Abies alba $\mathrm{A}$ & 1 & . & 1 & . & . & . & . & . & . & . & . & . & . & . & . & . & . & . & 1 & I \\
\hline \multicolumn{21}{|l|}{ Ch. Artemisietea vulgaris } \\
\hline Urtica dioica & + & . & . & + & + & 1 & . & + & 1 & 2 & 1 & + & + & . & + & + & . & + & 1 & IV \\
\hline \multicolumn{21}{|l|}{ Others } \\
\hline Alchemilla sp. & + & . & . & . & + & . & + & + & + & + & + & . & + & + & + & . & . & + & . & III \\
\hline Rubus idaeus & . & . & + & + & . & + & . & 1 & . & + & + & + & . & + & . & 1 & + & . & + & III \\
\hline Geum rivale & . & . & . & . & . & . & + & + & + & . & . & + & + & + & + & . & + & . & + & III \\
\hline Plantago major & + & . & . & . & . & . & 1 & . & + & . & . & . & . & + & + & + & . & + & + & III \\
\hline Listera ovata & . & . & . & . & . & . & + & + & . & . & . & . & + & . & . & . & + & + & + & II \\
\hline Oxalis acetosella & + & 1 & . & . & . & . & . & . & . & . & . & + & . & . & . & 1 & . & 1 & 1 & II \\
\hline Salix silesiaca B & . & . & . & . & 1 & . & 1 & . & . & . & 1 & . & . & . & . & 2 & . & . & + & II \\
\hline Роа аппиа & . & . & . & . & . & . & . & + & + & . & . & . & . & . & . & + & + & . & . & II \\
\hline Epilobium sp. & . & . & . & . & + & . & . & + & . & . & + & . & . & + & . & . & . & . & . & II \\
\hline Fragaria vesca & . & . & . & . & . & . & . & . & . & . & . & . & . & . & . & . & + & + & + & I \\
\hline Galeopsis speciosa & + & . & . & . & + & + & . & . & . & . & . & . & . & . & . & . & . & . & . & I \\
\hline Parnassia palustris & . & . & . & . & . & . & + & . & . & . & . & . & + & . & . & . & + & . & . & I \\
\hline Sorbus aucuparia B & . & . & . & . & . & . & . & . & . & . & . & 1 & . & . & . & 1 & . & + & . & I \\
\hline Tussilago farfara & . & . & . & . & . & . & . & . & . & + & . & . & . & + & . & . & . & + & . & I \\
\hline Ajuga reptans & . & . & . & . & . & . & . & . & . & . & . & . & + & . & . & . & . & . & + & I \\
\hline Digitalis grandiflora & . & . & . & . & . & . & . & . & . & . & . & + & . & . & . & + & . & . & . & I \\
\hline Festuca sp. & . & . & . & . & . & . & + & . & . & . & . & . & . & + & . & . & . & . & . & I \\
\hline Frangula alnus B & . & . & . & . & . & . & . & . & . & . & . & . & . & . & + & . & 1 & . & . & I \\
\hline
\end{tabular}

Sporadic taxa: Ch. Adenostylion alliariae, Betulo-Adenostyletea, Aruncus sylvestris 13; Delphinium elatum 12; Cirsium waldsteinii 7; Ch. Fagetalia, Querco-Fagetea, Alnus incana B 3(1); Chrysosplenium alternifolium 11; Corylus avellana C 3; Fagus sylvatica A 19(2); Galium odoratum 1; Neottia nidus-avis 19; Prenanthes purpurea 1; Ranunculus lanuginosus 12; Veronica montana 14; Lunaria rediviva 6; Ch. Molinio-Arrhenatheretea, Arrhenatherum elatius 4; Cardamine pratensis 10; Mentha longifolia 4; Ch. Artemisietea vulgaris, Artemisia vulgaris 4; Rumex alpinus 6; Eupatorium cannabinum 3(1); Myosoton aquaticum 11; Others: Aconitum lasiocarpum 6; Briza media 14; Carex sp. 19; Melandrium rubrum 1; Mycelis muralis 2; Orobanche pallidiflora 5; Rosa sp. C 3; Salix silesiaca A 5(2); Salix sp. B 3; Scrophularia scopoli 5; Thymus pulegioides 14; Tofieldia calyculata 17; Valeriana simplicifolia 14 
Explanations: Number of relevé - Location (Latitude - N; Longitude - E; Altitude a.s.1. [m]), date. 1 - Trzy Korony (49²5'03.4”; $20^{\circ} 24^{\prime} 46.7^{\prime \prime} ; 830$; Exposure: NE; Inclination: $\left.30^{\circ}\right)$, 30.07.2009; 2 - Sobczański g. (49²4’48”; 20²4’19”; 615; Exposure: SW; Inclination: 20), 19.08.2012; 3 - Szczawnica (49²5’00.9”;

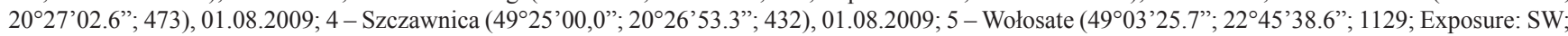

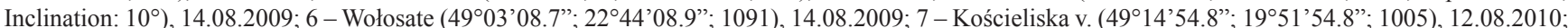
8 - Kościeliska v. (49¹5’18”; 1952'03.1”; 947), 12.08.2010; 9 - Chochołowska v. (49¹6’29.6”; 1949’30.8”; 909), 12.08.2010; 10 - Chochołowska v.

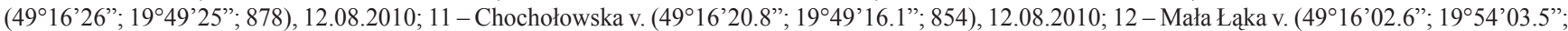
1100), 22.07.2009; 13 - Mała Łąka v. (49¹6’11.7”; 1954’01.9”; 1028), 22.07.2009; 14 - Mała Łąka v. (49¹6’14.7”; 1954’02.2”; 982), 22.07.2009; 15 -

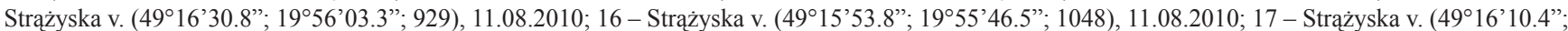
1955'54.3”; 965), 11.08.2010; 18 - Strążyska v. (49¹6’40.6”; 19॰56’15.6”; 925), 11.08.2010; 19 - Strążyska v. (49¹6’43.8”; 1956’25.1”; 887), 11.08.2010

contrast to $O$. flava, these species occurred in montane areas only very rarely and were scattered.

In the Carpathian Mts., butterbur broomrape is characteristic of the montane-belt Petsitetum kablikiani association of the Betulo-Adenostyletea class (Matuszkiewicz 2006), in which it reaches its occurrence optimum. However, it can be difficult to accept it as a characteristic species of the association. Butterbur broomrape is chiefly a species of the lower montane belt in Poland and its constancy does not exceed one degree. The highest degree of constancy was II and the species was proposed by Uziębło (2011) as a differentiating species of the association. It was found less frequently in tall herbs Petasitetum albi, PhalaridoPetasitetum hybridi or in ecotones of the Carpathian alder forest Alnetum incanae, riparian willow forest Salicetum albo-fragilis, with a contribution of species of Querco-Fagetea and Vaccinio-Piceetalia, and also Molinio-Arrhenatheretea classes. It occurred in large groups at the majority of the localities, often forming impressive clumps of more than a few dozen shoots.

Orobanche flava usually flowers from the second half of June onwards, reaching the optimum in July, sometimes even until the end of August (September). The flowering is spread over time as montane terrains have a diversified relief, incline, insolation and phytocoenotic conditions.

In Central Europe, Orobanche flava parasitizes species of the family Compositae, mainly Petasites kablikianus and P. albus, less frequently P. hybridus.
It was incorrectly reported as a parasite of Tussilago, Achillea, Origanum, Thymus, or Anemone (Beck 1930). In Poland, it is mainly recorded on P. kablikianus, considered as a Carpathian subendemite, rarely on $P$. albus (perhaps also on hybrid species) and $P$. hybridus. It is difficult to identify the host of the family Petasites due to its early flowering period.

All species of the Orobanche genus are strictly protected in Poland (Regulation 2012). O. flava was considered to be critically endangered (CR) in the Opole province (Nowak 2002). Majority of montane localities of $O$. flava in Poland are in national parks where its populations are in good condition. Some sites are outside protected areas and are susceptible to human pressure. Excessive trampling, gravel exploitation, community fragmentation, succession of tall herb and tree vegetation and strong shading, water course management, excessively frequent flooding and erosion of river channels pose threats to the communities and to the species. While $O$. flava should be protected, its hosts of the Petasites genus and alluvial communities of river valleys, sensitive to anthropopressure and natural factors should be monitored.

Acknowledgements. The author thanks the curators of the herbaria and other persons who made their data regarding the species available and helped with the field investigations. This work was supported by the Polish State Committee for Scientific Research (KBN grant no. NN303357733 (2008-2009) and NN303551939 (2010-2013).

\section{References}

Bartoszek W. \& Piwowarczyk R. 2008. Zaraza wielka. Orobanche elatior Sutton. In: Z. MireK \& H. PięKoś-Mirkowa (eds.). Czerwona Księga Karpat Polskich. Rośliny naczyniowe, pp. 316-318. W. Szafer Institute of Botany, Polish Academy of Science, Kraków.

Beck G. 1890. Monographie der Gattung Orobanche. Bibl. Bot. 19, 275 pp. Theodor Fischer, Cassel.
BЕск G. 1922. Orobancheae novae. Feddes Repertorium 30(1): 33-40

Beck G. 1930. Orobanchaceae. In: A. Engler (ed.). Das Pflanzenreich IV. (261), 348 pp. Verlag von Wilhelm Engelmann, Leipzig.

BiAŁecka K. 1982. Rośliny naczyniowe grupy Pilska w Beskidzie Żywieckim. Zesz. Naukowe UJ, Prace Bot. 10: 1-149. 
Dajdok K., Kącki Z., NowaK A., NowaK S. \& SpaŁeK K. 1998. Atlas rozmieszczenia rzadkich roślin naczyniowych w województwie opolskim. 204 pp. Uniwersytet Opolski, Opole.

EMberger L. 1935-36. Materiaux pour la Flore Marocaine VI. Bull. Soc. Sc. Nat. Maroc 15: 188-226.

FIEK E. 1881. Flora von Schlesien preussischen und österreichischen Antheils, enhaltend die wildwachsenden, verwilderten und angebauten Phanerogamen und Gefäss-Cryptogamen. 164+571 pp. J. U. Kern's Verlag, Breslau.

GierczyK B. \& Soboń J. 2008. Nowe stanowiska chronionych, zagrożonych i rzadko spotykanych gatunków roślin naczyniowych w Polsce. Przegląd Przyrodniczy 19(3-4): 19-31.

Grintescu G. P. 1945a (publ. 1946). Note despre Orobanche flava Martius. Notes on the Orobanche flava (Summary). Bulletin du Jardin et du Musée Botaniques de l'Université de Cluj, Roumanie 25(3-4): 150-152.

Grintescu G. P. 1945b. De Orobanche flava Martius. Bulletin du Jardin et du Musée Botaniques de l'Université de Cluj, Roumanie 26(1-2): 184.

GrodzIŃSKA K. 1976. Rośliny naczyniowe Skalic Nowotarskich i Spiskich (Pieniński Pas Skałkowy). Fragm. Flor. Geobot. 22(1-2): 43-127.

GrodzińsKa K. \& PANCER-Kotejowa E. 1960. Flora Wzniesienia Gubałowskiego. Monogr. Bot. 11(1): 1-196.

Guzikowa M. 1977. Rośliny naczyniowe Działów Orawskich i Bramy Sieniawskiej (południowa część Beskidu Żywieckiego). Monogr. Bot. 53: 1-267.

HalamsKi A. T. 2005. Orobanche lucorum zawleczona do Ogrodu Botanicznego UW. Biuletyn Ogr. Bot. 14: 115-117.

Holmgren P. K. \& Holmgren N. H. 1998. Index Herbariorum. New York Botanical Garden; http://sciweb.nybg.org/ science2/IndexHerbariorum.asp.

JASIEwICZ A. 1965. Rośliny naczyniowe Bieszczadów Zachodnich. Monogr. Bot. 20: 1-339.

KornAś J. 1957. Rośliny naczyniowe Gorców. Monogr. Bot. 5: $1-260$.

Kornaś J. \& MEdweckA-Kornaś A. 1967. Zespoły roślinne Gorców. I. Naturalne i na wpół naturalne zespoły nieleśne. Fragm. Flor. Geobot. 13(2): 167-316.

KotońsKa B. 1991. Rośliny naczyniowe Beskidu Małego (polskie Karpaty Zachodnie). Zeszyty Naukowe UJ, Prace Bot. 23: 1-199.

Kotula B. 1881. Spis roślin naczyniowych z okolicy Przemyśla. Spraw. Kom. Fizjogr. AU 15: 1-90.

Kotula B. 1883. Spis roślin naczyniowych z okolic górnego Strwiąża i Sanu z uwzględnieniem pionowego zasięgu gatunków. Spraw. Kom. Fizjogr. AU 17: 105-243.

Kreutz C. A. J. 1995. Orobanche. The European broomrape species. Central and northern Europe. Natuurhistorisch Genootschap, Limburg.

Matuszkiewicz W. 2006. Przewodnik do oznaczania zbiorowisk roślinnych Polski. In: J. B. FAliński (ed.). Vademecum Geobotanicum 3, 537 pp. Wyd. Nauk. PWN, Warszawa.

Meusel H., Jäger E., Rauschert S. \& Weinert E. 1978. Vergleichende Chorologie der zentraleuropäischen
Flora. II. Text xi+418 pp., Karten pp. 259-421. Gustav Fischer Verlag, Jena.

MichalewsKa A. 2004. Nowe stanowisko Orobanche flava (Orobanchaceae) w dolinie Sanu (Kotlina Sandomierska). Fragm. Flor. Geobot. Polonica 11(2): 424-426.

Mirek Z., Musią L. \& Wójcicki J. J. 1997. Polish herbaria. Polish Bot. Stud. Guideb. Ser. 18: 1-110.

Mirek Z. \& Piękoś-Mirkowa H. 1987. Flora synantropijna Kotliny Zakopiańskiej. Stud. Nat. Ser. A 30: 1-182.

Mirek Z., Piękoś-Mirkowa H., Zając A. \& Zając M. 2002. Flowering plants and pteridophytes of Poland. A checklist. In: Z. MireK (ed.). Biodiversity of Poland, 1, 442 pp. W. Szafer Institute of Botany, Polish Academy of Sciences, Kraków.

Nejfeld P. \& BartoszeK W. 2008. Zaraza drobnokwiatowa Orobanche minor Sm. In: Z. MireK \& H. PięKoś-Mirkowa (eds.). Czerwona Księga Karpat Polskich. Rośliny naczyniowe, pp. 319-321. W. Szafer Institute of Botany, Polish Academy of Science, Kraków.

Nikel A. 2006. Flora i zagadnienia geobotaniczne Pogórza Spiskiego. Fragm. Flor. Geobot. Polonica Supplementum 8:1-319.

NowaK A. 2002. Zaraza żółta - Orobanche flava Mart. ex F. W. Schultz. In: A. NowaK \& K. SpaleK (eds.). Czerwona księga roślin województwa opolskiego. Rośliny naczyniowe wymarłe, zagrożone i rzadkie, p. 142. Opol. Tow. Przyj. Nauk, Opole.

Nowak J. T., Gawryś W. \& Marcinkowski J. 2000. Index plantarum of outdoors cultivated herbaceous plants in Poland. Prace Ogrodu Bot. Uniw. Wrocł. 6(1), 399 pp. Wyd. Uniw. Wrock., Wrocław.

Pacyna A. 2004. Rośliny naczyniowe wschodniej części Pogórza Wielickiego i przylegającej części Beskidów (Karpaty Zachodnie). Prace Bot. UJ 38: 1-367.

PaWŁowski B. 1925. Geobotaniczne stosunki Sądecczyzny. Prace Monogr. Komis. Fizjogr. PAU 1: 1-336.

PawŁowski B., SokoŁowski M. \& Wallisch K. 1928. Zespoły roślin w Tatrach. Część VII. Zespoły roślinne i flora doliny Morskiego Oka, Rozpr. Wydz. Mat. Przyr. Dział A/B, 27: 171-311.

Piwowarczyk R. 2006. Róża francuska Rosa gallica L. na Przedgórzu Iłżeckim (Wyżyna Małopolska). Chrońmy Przyr. Ojcz. 62: 55-60.

Piwowarczyk R. 2010. Rośliny naczyniowe wschodniej części Przedgórza Iłżeckiego (Wyżyna Małopolska). Prace Bot. UJ 43: 1-344.

Piwowarczyk R. 2011. Orobanche mayeri (Suess. \& Ronniger) Bertsch \& F. Bertsch - the new species to Poland. Acta Soc. Bot. Pol. 80(3): 179-183. DOI: http://dx.doi. org/10.5586/asbp.2011.020

Piwowarczyk R. 2012a. The genus Orobanche L. (Orobanchaceae) in the Małopolska Upland (S Poland): distribution, habitat, host preferences and taxonomic problems. Biodiv. Res. Conserv. 26: 3-22. DOI:10.2478/ v10119-012-0009-2

PiwowARCzyK R. 2012b. Orobanche alba subsp. alba and subsp. major (Orobanchaceae) in Poland: current distribution, taxonomy, plant communities, hosts, and seed micromorphology. Biodiv. Res. Conserv. 26: 23 38. DOI:10.2478/v10119-012-0005-6 
PiwOWARCZYK R. 2012c. Revised distribution and plant communities of Orobanche alsatica and notes on the Orobanchaceae series Alsaticae in Poland. Biodiv. Res. Conserv. 26: 39-51. DOI:10.2478/v10119-012-0008-3

Piwowarczyk R. 2012d. A revision of distribution and the ecological description of Orobanche picridis (Orobanchaceae) at the NE limit of its geographical range from Poland and Ukraine. Acta Agrobot. 65(1): 91106. DOI: http://dx.doi.org/10.5586/aa.2012.047

PiwowARCZYK R. 2012e. Orobanche bohemica Čelak. (Orobanchaceae) at the eastern limit of its geographical range: new data on its distribution in Poland. Biodiv. Res. Conserv. 26: 53-59. DOI: 10.2478/v10119-0120007-4

Piwowarczyk R. 2012f. Revised distribution and phytosociological data of Orobanche coerulescens Stephan in Willd. (Orobanchaceae): Poland in relation to Central Europe. Biodiv. Res. Conserv. 26: 61-72. DOI: 10.2478/v10119-012-0007-4

PiwowARCZyK R. 2012g. Orobanche purpurea (Orobanchaceae) in Poland: current distribution, taxonomy, plant communities, and preferred hosts. Biodiv. Res. Conserv. 26: 73-81. DOI: 10.2478/v10119-012-0006-5

PiwowARCZyK R. 2012h. A revision of distribution and historical analysis of preferred hosts of Orobanche ramosa (Orobanchaceae) in Poland. Acta Agrobot. 65(1): 53-62. DOI: http://dx.doi.org/10.5586/aa.2012.043

Piwowarczyk R. 2013. Seed productivity in relation to other shoot features for endangered parasitic plant Orobanche picridis F. W. Schultz (Orobanchaceae). Pol. J. Ecol. 61(1): 55-64.

PiwowARCZyK R. 2014. Orobanche caryophyllacea (Orobanchaceae) in Poland: current distribution, taxonomy, plant communities and hosts. Acta Agrobot. 67(3):97118. DOI: 10.5586/aa.2014.035.

Piwowarczyk R., Chmielewski P. \& Cwener A. 2011. The distribution and habitat requirements of the genus Orobanche L. (Orobanchaceae) in SE Poland. Acta Soc. Bot. Pol. 80(1): 37-48. DOI: http://dx.doi. org/10.5586/asbp.2011.006

Piwowarczyk R., Chmielewski P., Gierczyk B., PiwowarSKi B. \& Stachyra P. 2010. Orobanche pallidiflora Wimm. \& Grab. in Poland: distribution, habitat and host preferences. Acta Soc. Bot. Pol. 79 (3): 197-205. 205. DOI: http://dx.doi.org/10.5586/asbp.2010.025

PiwowarczyK R. \& Kirpluk I. 2011. Orobanche flava (Orobanchaceae) w Ogrodzie Botanicznym Uniwersytetu Warszawskiego. Fragm. Flor. Geobot. Polonica 18(1): 163-165.

Piwowarczyk R., Nobis M. \& Przemyski A. 2009. Orobanche bartlingii Griseb. (Orobanchaceae) in Poland: taxonomical position, distribution and habitat requirements. Biodiv. Res. Conserv. 13: 3-8. DOI:10.2478/v10119009-0001-7

Piwowarczyk R. \& Przemyski A. 2009. New locality of Orobanche coerulescens Stephan ex Willd. (Orobanchaceae) at the NW limit of its geographical range. Acta Soc. Bot. Pol. 78 (4): 291-295. DOI: http://dx.doi. org/10.5586/asbp.2009.038

PiwowarczyK R. \& PrZemyski A. 2010. The distribution and habitat preferences of the declining species Orobanche arenaria (Orobanchaceae) at the northern limit of its geographical range. Acta Soc. Bot. Pol. 79(1): 43-50. DOI: http://dx.doi.org/10.5586/asbp.2010.007

Pusch J. \& Günther K.F. 2009. Orobanchaceae (Sommerwurzgewächse). In: G. Hegi (ed.). Illustrierte Flora von Mitteleuropa Bd. 6/1A, Lieferung 1, 99 pp. Weissdorn-Verlag, Jena.

Rätzel S. \& Uhlich H. 2004. Orobanche benkertii sp. nov. (Orobanchaceae Vent.) und weitere Orobanche-Sippen aus dem Nordwest-Kaukasus. Feddes Repertorium 115(1-2): 189-211.

Regulation of the Minister of the Environment of 5 January 2012 on wild species of plants under protection. Journal of Laws No 14, item 81.

RuMSEY J. \& JuRY S. L. 1991. An account of Orobanche L. in Britain and Ireland. Watsonia 18: 257-295.

SchuBE T. 1903. Verbreitung der gefässpflanzen in Schlesien preussischen und österreichischen Anteils. R. Nischowsky, Breslau.

Stuchlik L. 1968a. Zbiorowiska leśne i zaroślowe pasma Policy w Karpatach Zachodnich. Fragm. Flor. Geobot. 14(4): 441-484.

STUChlik L. 1968b. Zbiorowiska ziołoroślowe i źródliskowe pasma Policy w Karpatach Zachodnich. Fragm. Flor. Geobot. 14(4): 485-494.

Stuchlikowa B. \& Stuchlik L. 1962. Geobotaniczna charakterystyka pasma Policy w Karpatach Zachodnich. Fragm. Flor. Geobot. 8(2): 229-396.

Towpasz K. 1975. Rośliny naczyniowe południowo-wschodniej części Beskidu Wyspowego. Monogr. Bot. 48: 1-146.

TyszKowski M. 1993. Zaraza żółta Orobanche flava w Sudetach. Chrońmy Przyr. Ojcz. 49(2): 87-89.

Uechtritz R. 1865. Nachträge zur schlesischen Flora (IV). Verhandl. Bot. Ver. Prov. Brandenburg und angr. Länder 7: 72-105.

Uhlich H., Pusch J. \& Barthel K. J. 1995. Die Sommerwurzarten Europas: Gattung Orobanche. 235 pp. Westarp-Wiss., Magdeburg.

UzięBŁo A. K. 2011. Petasites kablikianus Tausch ex Berchold as a pionier species and its abilities to colonie initial habitats. Wydawnictwo Uniwersytetu Śląskiego, Katowice.

WAYDA M. 2001. Rośliny naczyniowe północnej części Okręgu Radomyskiego (Kotlina Sandomierska). Prace Bot. UJ 36: 1-117.

ZAJĄC A. 1978. Założenia metodyczne "Atlasu rozmieszczenia roślin naczyniowych w Polsce”. Wiad. Bot. 22(3): 145-155.

ZaJĄC A. \& ZajĄC M. (eds.). 2001. Distribution Atlas of Vascular Plants in Poland. xii+714 pp. Edited by Laboratory of Computer Chorology, Institute of Botany, Jagiellonian University, Cracow.

ZARZYCKI K. 1956. Zarastanie żwirowisk Skawicy i Skawy. Fragm. Flor. Geobot. 2(1): 111-142.

ZARZYCKI K. 1981. Rośliny naczyniowe Pienin. Rozmieszczenie i warunki występowania. 257 pp. PWN, Instytut Botaniki PAN, Kraków-Warszawa.

ZÁzvorka J. 1997. Orobanchaceae Vent. Zárazovité. In: K. Golí́šovÁ (ed.). Flóra Slovenska 5(2): 460-529. VEDA, Bratislava. 
ZÁzvorka J. 1999. Zarazá rusá (Orobanche flava) v Krkonošich. Butterbur Broomrape (Orobanche flava) in the Giant Mts. Opera Corcontica 36: 215-218.

Zemanek B. 1989. Rośliny naczyniowe Bieszczadów Niskich i Otrytu (polskie Karpaty Wschodnie). Prace Bot. UJ 20: $1-185$.
ZemaneK B. \& WinNicki T. 1999. Rośliny naczyniowe Bieszczadzkiego Parku Narodowego. Monogr. Bieszczadzkie 3: 1-249.

ZuBrzyCKi J. 1894. Flora Pienin. Rośliny naczyniowe. Spraw. Komis. Fizjogr. AU 29: 70-95. 


\section{Appendix 1. List of localities of Orobanche flava in Poland}

Symbols and abbreviations: app. - approximately, ATPOL - Distribution Atlas of Vascular Plants in Poland database, com. - commune, distr. - district, leg. - collected by; n. - near, P. - Petasites, phot. - photography, res. - reserve, v. - valley, vid. - seen by, ? - doubtful locality

BE: 95 - Kuźnica (Góry Sowie Mts), (after ATPOL); BF: 05 - Masyw Słonecznej massif (Góry Sowie Mts), on P. albus, (Uechtritz 1865; Beck 1890, 1930; Fiek 1881; Schube 1903); Góry Sowie Mts (Eulengebirge, Sovi hory), leg. H. Wolff 08.1871 (B); Góry Sowie Mts, N-side of Sonnenkoppe Mt, leg. H. Wolff 07.1893 (B); Sonnenkoppe, leg. E. Zilk 26.07.1870 (GLM, 138865); CF: 38 - Stara Kuźnia, (Dajdok et al. 1998, after ATPOL); 41 - Podlesie, 1 km SES of Podlesie (Tyszkowski 1993); DF:90 - Wierzbina Forest, Cieszyn Marklowice, 4946’33'N, 18³6'21'”, on P. hybridus, (<10), vid. M. Fiedor, 29.06.2014; 96 - Jaworzyna (Kotońska 1991); Mucharz (Kotońska 1991); 97 - Mucharz-Upalisko (Kornaś J., unpubl., 1968, after ATPOL); Mucharz-Upalisko, 290-230 m (Kotońska 1991); Mucharz-Upalisko, 295 m, opposite Zagórze (Kotońska 1991); DG: 01 - Beskid Śląski Mts, Mała Czantoria, on P. albus, 4941'20.9'N, 1844'59.6”E, 374 m, leg. R. Piwowarczyk, 17.07.2009 (KTC); 06 - Western Carpathian Mts, Beskid Makowski Mts, Cicha n. Koszarawa, on P. sp., on the right bank of the Koszarawa near an asphalt road, very abundant, 635 m, leg. W. Bartoszek, 09.07.2001 (0258292, 0258293, 0258294); Koszarawa - Cicha, Koszarawa stream v., near an asphalt road to the terminus of the Koszarawa-Jałowiec coach service, among P. kablikianus, 670 m, leg. W. Bartoszek, 22.06 .1998 (KRA, 0260554, 0260555); Koszarawa - Cicha (above a Koszarawa - Jałowiec bus stop), by an asphalt road and green trail, $675 \mathrm{~m}$, on P. kablikianus, leg. W. Bartoszek, 15.04.1998 (KRA, 0260559); Lachowice, 440 m (Bartoszek W., unpubl., 1998, after ATPOL); Koszarawa, A. Mielnikow 06.07.1992, phot., http://www.atlas-roslin.pl/gatunki/Orobanche flava.htm; 07 - n. Maków Podhalański (Zarzycki 1956); Maków Podhalański, 355 m (Bartoszek W., unpubl., 1998, 2000, 2001; Hucisko - Przysłop (Anielska T., unpubl., 1989, after ATPOL); Juszczyn on the Skawica (Stuchlik 1968b); Białka, Skawica v. (Bartoszek W., unpubl., 2003, after ATPOL); Skawica, Skawica Sołtysia v. (Bartoszek W., unpubl., 2004, after ATPOL); 08 - Juszczyn, Skawica river mouth, 370-375 m (Bartoszek W., unpubl., 1999, 2000, after ATPOL); 13 - Leśna v. (Kornaś J., unpubl., 1968, after ATPOL); Cisiec, 410 m (Białecka 1982); Węgierska Górka, 405 m (Białecka K., unpubl., 1983, after ATPOL); 14 - Wieprz, Soła v., 380 m, in riparian thickets, on $P$. hybridus, leg. K. Białecka, 01.07.1970 (KRA, 109633); Wieprz, 380 m (Białecka 1982); Wieprz, Radziechowy-Wieprz com., Bielsko-Biała province, floodplain thickets, leg. M. Skolarz, 29.06.1987 (KTU, 41263); Beskidy Zachodnie Mts, Góra Grojec n. Żywiec on the Jaz: 21e, 358 m, alder grove on gravel bank, leg. K. Nowak, 07.08.1992 (KTU, 99026); Juszczynka, $440 \mathrm{~m}$ (Białecka 1982); 15 - Beskid Makowski Mts, Pewel Mała n. Żywiec, in riparian shrubs (Alnetum incanae), on right floodplain terrace of the Koszarawa n. Pewel Mała - Skrzyż bus stop, on P. kablikianus, 390 m, leg. W. Bartoszek, 06.07.1998 (KRA, 0260553); Pewel Mała (Kornaś J., unpubl., 1968, after ATPOL); Jeleśnia, Gajka forest (Białecka K. unpubl., 1971, after ATPOL); Gajka, 480 m, 500 m (Białecka 1982); Sopotnia, 650 m (Białecka 1982); Pewel Mała, 390 m (Bartoszek W., unpubl., 1998, after ATPOL); Jeleśnia, on the Sopotnia river (Bartoszek W., unpubl., 1998, after ATPOL); Koszarawa, 585-595 m (Bartoszek W., unpubl., 1999, after ATPOL); Pewel Mała, junction towards Koszarawa, 390 m (Bartoszek W., unpubl., 1998, after ATPOL); 16 - Widły at the foot of Mt Babia Góra, on meadow margin, leg. J. Błaszczyk, 06.1964 (KRA, 94598); Zawoja, on Marków stream at the foothills of Mt Babia Góra, leg. K. Kostrakiewicz, 15.07.1949 (KRAM, 230104); Zawoja, forest margin, P. thickets, leg. W. Bąba, 06.09.1992 (KTU, 45892); Zawoja, forest margin, leg. B. Chowaniec, 06.09.1997 (KTU, 64205); Zawoja Widły, roadside tall herbs near fence, leg. A. Sendek, 10.09 .1982 (KTU, 28210); Zawoja, thickets by the river, leg. J. Jazowiecka, 03.09.1997 (KTU, 64206); Zawoja, thickets, leg. G. Porchańska, 03.09.1997 (KTU, 64207); Zawoja, leg. K. Borek, 09.1997 (KTU, 64209); Zawoja, roadside thickets, leg. A. Zaręba, 09.09.2000 (KTU, 64208); Zawoja, parasitic on P. sp., roadside, leg. B. Chwastowski, 15.07.1975 (CHRZ); Zawoja (Bartoszek W., unpubl., 2003, after ATPOL); Zawoja Widły, 600 m, (Stuchlikowa \& Stuchlik 1962); Jaworzyna, 785 m, (Stuchlikowa \& Stuchlik 1962); on the Błędna, $680 \mathrm{~m}$ (Stuchlikowa \& Stuchlik 1962); N slope of Mt Lachów Groń, 740-750 m (Anielska T., unpubl., 1989, after ATPOL); Cicha, 710 m (Anielska T., unpubl., 1989, after ATPOL); Mt Babia Góra (Beck 1890); Babia Góra, leg. ?, 24.07.1906 (KRAM, 244726); 17 - Pasmo Policy ridge, Biała, leg. B. Stuchlik \& L. Stuchlik, 1995 (KRA, 0249978); Zimna Dziura, 720 m (Stuchlikowa \& Stuchlik 1962); Mosorny Groń, 915 m (Stuchlikowa \& Stuchlik 1962); Policzne, 720 m (Stuchlikowa \& Stuchlik 1962); Zubrzyca Górna, 780 m (Stuchlikowa \& Stuchlik 1962); Zawoja - Policzna, towards Krowiarki (Stuchlik 1968b); Sucha Góra E (Stuchlik 1968a); 22 - Przysiółek Stańcówka (Herzog B., 1987, after ATPOL); Rajcza, by stream, leg. M. Ciołek, 20.07.1988 (KTU, 44365); Rajcza (Kornaś J., unpubl., 1968, after ATPOL); Ujsoły, 530 m (Białecka 1982); 23 - Milówka, thickets, leg. I. Hola, 16.09.2000 (KTU); 24 - Złatna, 720-750 m (Białecka K., unpubl., 1971, after ATPOL); Bystra river v., Złatna, 560 m (Białecka 1982); Kościelec, 750 m (Białecka 1982); Straceniec, 700 m (Białecka 1982); 27 - Zubrzyca Górna, 780 m (Guzikowa 1977); 33 - Rycerka stream v., 650 m (Kornaś J., unpubl., 1968, after ATPOL); Rycerka (Herzog B., 1987, after ATPOL); E of Racza v. (Kornaś J., unpubl., 1968, after ATPOL); Sól stream v. (Herzog B., 1987, after ATPOL); 34 - Beskid Żywiecki Mts (Żywiec), Soblówka, Petasitetum kablikiani, leg. H. Piękoś \& B. Bzowska, 11.07.1970 (G, 46808; KRA, 70548; KRAM, 097360; KTU 10559; LBL; LE; LOD, 154409; POZ); Soblówka (Kornaś J., unpubl., 1968, after ATPOL); Grupa Pilska, Dolina Straceńca v., in alder grove, 700 m, leg. K. Białecka, 01.08.1971 (KRA, 109632); Oszust, NW (Kornaś J., unpubl., 1966, after ATPOL); Glinka v., 680 m (Białecka 1982); 48 - Witow, 890 m (Grodzińska \& Pancer-Kotejowa 1960); 49 - Zagrody, 830 m (Grodzińska \& Pancer-Kotejowa 1960); Rabanisko, 880 m (Grodzińska \& Pancer-Kotejowa 1960); 57 - Tatry, Dolina Strążyska v. (Piękoś-Mirkowa H. \& Mirek Z., unpubl., 1989, after ATPOL); 58 - Dolina Chocho-

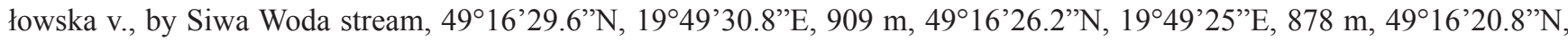


1949’16.1”E, 854 m, 49¹6’11.2”N, 1949’07’E, 947 m, leg. R. Piwowarczyk, 12.08.2010 (KTC); 59 - Tatra Mts, Hala Kirowa in Dolina Kościeliska v., by stream, on P. albus, leg. A. Żmuda, 20.08.1910 (KRAM, 494742; WA, 28415); Dolina Kościeliska v. n. forester's lodge, among P. albus, in masses, 920 m, leg. B. Pawłowski, 23.07.1923 (KRAM, 305102); Dolina Kościeliska v., by stream, 900 m, on P. albus, leg. B. Pawłowski, 23.07.1923 (WA, 28416); on the Czarny Dunajec river, n. Dolina Kościeliska v., behind sawmill, in an agglomeration of $P$., leg. J. Walas, 09.07.1932 (KRA, 0125672); Kiry n. Zakopane, on the bank of the Kirowa Woda, on P. kablikianus, leg. J. Mądalski, 21.08.1965 (KRAM, 494746); Dolina Kościeliska v., towards its end, on P. glabrata, 925 m, leg. B. Pawłowski, 10.07.1936 (KRAM, 305101); Kościelisko n. Zakopane, on the left bank of the Kirowa Woda, n. hostel, leg. J. Mądalski, 25.08.1938 (KRAM, 494751); Kościelisko, between Kiry and Brama Kontaka villages, among $P$. thickets, by the road, leg. ?, 07.08.1955 (KRAM, 494747, 494748, 494749, 494750); Dolina Kościeliska v., tall herbs by stream, leg. A. Pacyna, 15.07.1960 (KRA, 0121702); Dolina Kościeliska v., on P., leg. M. Ceynowa, 17.08.1960 (TRN); Dolina Kościeliska v., tall herbs, leg. A. Sendek, 16.08.1986 (KRA, 0138565); by Czarny Dunajec river, between Siwa/Kirowa Woda confluence and Kajsówka, leg. J. Płazińska, 01.07.1981 (KRAM, 290326); Dolina Kościeliska v., P. scrub by Kirowa Woda and Kościelski Potok streams, Brama Kontaka, Cudakowa Polana, 49¹4'54.8’N,

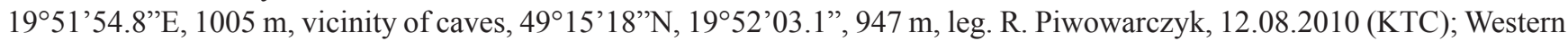
Tatra Mts, Dolina Ku Dziurze v., 896 m, leg. R. Rajchel, 13.08.1960, 25.08.1961 (KRA, 0112679, 0121701); Pogórze Gubałowskie, thickets by Czarny Dunajec river, on P. albus, leg. G. Pancer, 26.08.1955 (KRA, 0176200); Western Tatra Mts, end of Dolina Spadowiec v., by "Pod Reglami" route, 920 m, leg. H. Tacik, T. Tacik, 19.08.1978 (KRAM, 386088); Tatra Mts,

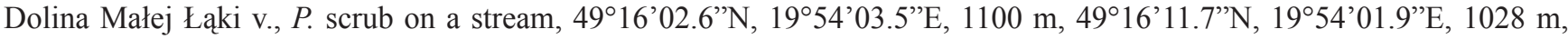

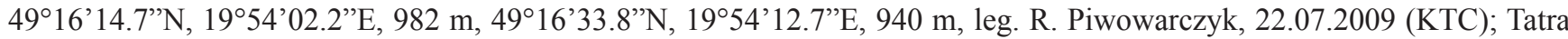
Mts, Dolina Strążyska v., P. scrub by stream, on P. kablikianus, $49^{\circ} 16^{\prime} 30.8^{\prime \prime} \mathrm{N}, 19^{\circ} 56^{\prime} 03.3^{\circ} \mathrm{E}, 929 \mathrm{~m}$, at the foot of Polana Strążyska meadow, $49^{\circ} 15^{\prime} 53.8^{\prime}$ 'N , 19 $9^{\circ} 5^{\prime} 46.5^{\prime} \mathrm{E}, 1048 \mathrm{~m}$, roadside on scree margins n. Trzy Kominy ridge, 49 $16^{\prime} 10.4^{\prime \prime} \mathrm{N}$,

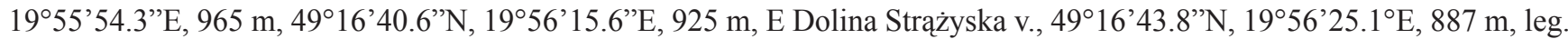
R. Piwowarczyk, 11.08.2010 (KTC); Roztoki, 870 m, (Grodzińska \& Pancer-Kotejowa 1960); Ubocz (Kotlina Zakopiańska), (Mirek \& Piękoś-Mirkowa 1987); Tatra Mts: Przyslop Miętusi, (A. Mielnikow 29.06.2002, phot., phot., http://www.atlas-roslin.pl/gatunki/Orobanche_flava.htm; ED: 37 - Botanical Garden, Warsaw, on P. hybridus, leg. Kirpluk I. \& Piwowarczyk R., 10.07.2009 (KTC), (Piwowarczyk \& Kirpluk 2011); EF: 74 - cfr., Okocim, leg. Polański (KRAM, 202186); EG: 04 Biała Woda on the Dunajec (Szewczyk M., unpubl., 1998, after ATPOL); 05 - Rdziostów (Pawłowski 1925); 11 - Gorce Mts, Rzeki, left bank of the Kamienica n. Gajówka, on P. hybridus, 690 m, leg. A. Kornaś \& J. Kornaś, 21.07 .1950 (KRA, 0121710); Szczawa, Kamienica gravel pits (Kornaś \& Medwecka-Kornaś 1967); Szczawa - Wiatrówki, 620 m, on P., 49³7’N, 20¹5’E, leg. J. Żelazny, 19.08.2005 (KRA, 0273863); Przysłup, Kamienica v., leg. K. Rostański, 29.06.1995 (KTU, 99139); at the foot of Mt Kudłoń, $830 \mathrm{~m}$ (Kornaś 1957); Gorce, at the foot of Mt Kudłoń, Petasites scrub, Petasitetum kablikiani, lower montane belt, leg. R. Piwowarczyk, 06.07.2005 (KTC); Rzeki, 690 m (Kornaś 1957); 12 - Szczawa, 525 m (Kornaś 1957); Szczawa, 490 m (Towpasz 1975); Beskid Wyspowy Mts, Szczawa, shrubs with P. hybridus, 490 m, leg. K. Towpasz, 07.07 .1968 (KRA, 82140, 0123258, 0123289); Kamienica, 480 m (Kornaś 1957); Białe, 610 m (Towpasz 1975); 14 - Podrzecze on the Dunajec, (Szewczyk M., unpubl., 1997, after ATPOL); Dolina Kamienicy v., in Rzeki, (Kornaś \& Medwecka-Kornaś 1967); on the Dunajec river n. Chełm (Nowy Sącz distr.), on P. hybridus, leg. B. Pawłowski, 12.08.1919 (KRAM, 305104); osiers on the Dunajec in the Chełm province, opposite Nowy Sącz, on P. hybridus, leg. B. Pawłowski, 21.07.1921 (KRAM, 305103); 22 - Ochotnica Dolna, 490 m (Kornaś 1957); 24 - Gaboń - Przehyba (Szewczyk M., unpubl. 1995, after ATPOL); 27 - Śnietnica n. Uście Gorlickie, n. Biała river, on P. hybridus, 49³0.8'69’N, 21³'7.28’E, vid. P. Kauzol, $2012 ; 31$ - Kramnica, 620 m (Grodzińska 1976); Jurgów, stream on Białka, 680-740 m (Grodzińska \& Pancer-Kotejowa 1960); Pieniński Pas Skałkowy belt, Kramnica, alder groves on the Białka, leg. K. Grodzińska, 07.07.1927 (KRAM, 259789); in alder grove, at the foot of a rock, 620 m, on P., leg. K. Grodzińska (KRAM, 219788); 32 - Kąty - Sromowce Wyżne, Dunajec gravel pits, leg. K. Zarzycki, 23.06.1967 (KRAM, 363768); Kąty, Dunajec alluvia (Zarzycki 1981); 33 - Dolina Pienińskiego Potoku v. (Zubrzycki 1894); Pieniny Mts - generally (Pawłowski 1925); Pieniński Potok stream, leg. J. Walas, 14.07.1936 (KRA, 0125353); Pieniny National Park, leg. P. Szatkowski (KRAM, 356939, as O. lutea); Pieniny Mts, Trzy Korony, leg. M. Sychowa, 06.08.1958 (KRAM, 205385); Pieniny Mts, at the foot of Trzy Korony, leg. T. Tacik, 04.08.1958 (KRAM, 386096); at the foot of Trzy Korony, by the trail, vid. R. Piwowarczyk, 19.08.2012; Wąwóz Sobczański gorge, 49²4'48’N, 20²4'19”E, 615 m, vid. R. Piwowarczyk, 23.07.2012; Pieniny Mts, on the Dunajec, Szczawnica, behind the confluence towards Sokolica Mt (30), on $P$. kablikianus, 49 $25^{\prime} 00.9^{\circ} \mathrm{N}, 20^{\circ} 27^{\prime} 02.6^{\prime \prime} \mathrm{E}, 473 \mathrm{~m}$, leg. R. Piwowarczyk, 01.08.2009; Dunajec alluvia; opposite Wysoka Ska-

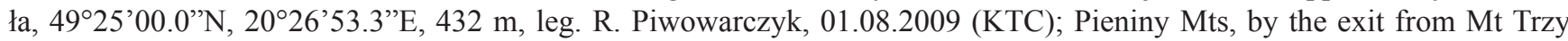
Korony towards Krościenko, by Przełęcz Szopka pass, roadside in fir forest (>100), on P. albus, 49²5'03.4'N, 20²4'46.7'”E, 830 m, leg. R. Piwowarczyk, 30.07.2009; “Za Kijowem” v., 500 m (Pawłowski 1925); 35 - n. Piwniczna (Pawłowski 1925); 36 - Jaworzyna, on a water course, S slope from the hostel, 780 m, leg. B. Kotońska, 16.07.1986 (KRA, 0272825); 41 - Dolina Białki v., on alluvia of the mouth of the Jaworowy Potok stream up to Mt Czarna Góra, on P. kablikianus, leg. J. Płazińska, 23.07.1981 (KRAM, 288200); NEE of S border of Łapszanka village, vicinity of so-called „Moczarka”, by the road with spruce woodland, 49 $21^{\prime} 06.6^{\prime} \mathrm{N}, 20^{\circ} 13^{\prime} 08^{\prime}$ 'E, $960 \mathrm{~m}$, vid. P. Niedźwiedzki, 02.01.2012; 42 - S of Stawiska village (S of Łapsze Niżne village), roadside in the forest, by beech grove and stream, n. Niebieska Dolina res., 49²1'47.6”'N, 20¹4’34.8”'E, 690-720 m, vid. P. Niedźwiedzki, 02.01.2012; Pogórze Spiskie, tall herb communities, on P. kablikianus, min. 525 m, EG: 31 , 32, 41, 42 (Nikel 2006); 46 - n. Muszyna (Pawłowski 1925); 50 - Tatra Mts, Mt Zawrat, leg. M. Ceynowa-Giełdon \& W. Gugnacka, 20.08.1968 (TRN); by road to lake Morskie Oko, na P., leg. R. Kobendza, 07.1939 (WA, 28417); Dolina Filipka 
v. (Piękoś-Mirkowa H. \& Mirek Z., unpubl., 1989, after ATPOL); 51 - Łysa Polana (Pawłowski et al. 1928); High Tatra Mts, Łysa Polana, in P., on stream on Slovak side, leg. A. Jasiewicz, 25.08.1965 (KRAM, 414014); Tatra Mts, Białka n. Jaworzyna, on P., leg. W. Wagner, 08.1889 (POZ); FF: 30 - Wola Mielecka, (Wayda M., unpubl., 1996, after ATPOL); 37 - SW of Kulno village (Michalewska 2004); 76 - Grzegorzówka Kanada, mixed forests (Krowiak M., unpubl., 2000, after ATPOL); 85 - Biskupice Melsztyńskie (Pacyna A., unpubl., 1992, after ATPOL; Pacyna 2004); 99 - by right tributary of Prałkowicki stream, on P. hybridus (Kotula 1881); FG: 12 - Wietrzno, in willow shrubs on the Jasionka, on P. kablikianus and P. hybridus, leg. K. Oklejewicz (KRA, 0141631); Zboiska n. Dukla, in thickets on the Jasionka, on P. kablikianus, leg. K. Oklejewicz, 24.08.1990 (KRA, 0142146); Równe Kopalnia, (Oklejewicz K., unpubl. 1989, after ATPOL); Równe Kopalnia (Oklejewicz 1992, after ATPOL); Wietrzno (Oklejewicz 1992); Zboiska (Oklejewicz 1992, after ATPOL); 14 - Besko, leg. Betkowski, 06.07.1961 (OPOL, P/7726); Besko (Oklejewicz K., unpubl., 1989, after ATPOL); 16 - Besko (Oklejewicz 1992, after ATPOL); 22 Beskid Niski Mts, Przełom Jasiołki gap, Tylawa (Krosno distr.) - Jasliska road (Sanok distr.), between Mt Piotruś and Mt Ostra, on P. hybridus, on margin on strem-side forest, 410 m, leg. A. Kornaś \& J. Kornaś, 20.07.1966 (KRA, 0176201); Jaśliska, Tylawa (Kornaś J., unpubl., 1966, after ATPOL); Góra Kamieniec, N part (Oklejewicz K., unpubl., 2000, after ATPOL); Cergowa Góra n. Dukla (Tyszkowski 1993); 33 - Daliowa, 400-450 m (Oklejewicz K., unpubl., 1997, after ATPOL); 34 - Góra Żebracza 727 m (Oklejewicz K., unpubl, 2000, after ATPOL); 48 - Hulskie (Zemanek 1989); g. 754, SW foothill, 480 m (Zemanek 1989); 57 - Dołżyca, 510 m (Jasiewicz 1965), Bieszczady Mts, Dolina Górnej Solinki v., vid. B. Gierczyk, 17.07.2008; 58 - Smerek, village, 600 m (Jasiewicz 1965); 59 - Smolnik, 650 m (Zemanek 1989); 67 - Bieszczady Zachodnie Mts, on the Smerek, by road towards Okraglik, leg. M. Pawlus, 07.07.1977 (KRAM, 257855, 257880, 257881); 68 - Moczarne n. Wetlina, in Tarnica v., on P. sp., leg. A. Jasiewicz, 13.07.1961 (KRAM, 414011); Moczarne, mouth of Beskidnik stream, hostel (Szewczyk M., unpubl., 1993, after ATPOL; Zemanek \& Winnicki 1999); Muchanin Wierch (Szewczyk M., unpubl., 1993, after ATPOL); Wetlina - Osada (Szewczyk M., unpubl., 1993, after ATPOL; Zemanek \& Winnicki 1999); Dolina Wielki Lutowy v., 720 m (Jasiewicz 1965); 69 - Bieszczady Zachodnie Mts, Ustrzyki Górne, 635 m (Jasiewicz 1965); Bieszczady Mts, ad pagum Bereżki, in associatione Petasitetum kablikiani, 610 m, leg. K. Zarzycki, 15.07.1961 (G, 38255; KRA, 70649; LE; POZ; W, 17872); Ustrzyki Górne - Berehy, roadside, on P. hybridus, leg. M. Kuc, 10.07.1982 (KRA, 0121709); Ustrzyki Górne, roadside, leg. M. Gasz, 26.07.2006 (KTU, 99024); Ustrzyki Górne, Rzeczyca river v., (Zemanek B., unpubl., 1995, after ATPOL; Zemanek \& Winnicki 1999); Ustrzyki Górne, park settlement (Zemanek B., unpubl., 1994, after ATPOL); Ustrzyki Górne, forest settlement (Zemanek B., unpubl., 1995, after ATPOL); Ustrzyki Górne, Terebowiec stream v. (Mitka J. \& Korzeniak J., unpubl., 1994, after ATPOL); Terebowiec stream v., middle and S part (Zemanek B., unpubl., 1993, after ATPOL; Zemanek \& Winnicki 1999); Zwór n. Bereżki, 620 m (Jasiewicz 1965); Bereżki, in associatione Petasitetum kablikiani, 610 m, leg. K. Zarzycki, 15.07.1961 (LOD, 154408); Bereżki, in Alnetum incanae, on the right bank of the Wołosaty river, leg. K. Zarzycki, 15.07.1961 (KRAM, 423110); alder groves between Ustrzyki Górne and Bereżki, 620 m, leg. A. Jasiewicz, 22.07.1956 (KRAM, 414012); Bereżki, alder grove on the right side of Bereżki, $620 \mathrm{~m}$, leg. A. Jasiewicz, 06.08.1960 (KRAM, 414013); Bereżki, 600-630 m (Jasiewicz 1965); Nasiczniański stream, middle part (Szewczyk M., unpubl., 1993, after ATPOL; Zemanek \& Winnicki 1999); 79 - Wołosate, 780 m (Jasiewicz 1965); Buczyna on the Włosatka, 870 m, on $P$. albus (Kotula 1883); GG: 60 - Terebowiec stream v., end of road, vicinity of a quarry (Zemanek B., unpubl., 1994, after ATPOL; Zemanek \& Winnicki 1999); Terebowiec stream v., N slope of Szeroki Wierch (Zemanek B., unpubl., 1993, after ATPOL); Bieszczady Zachodnie Mts, Muczne, meadow, leg. M. Quelec, 12.07.2001 (Herbarium M. Wołkowycki, 4796); 70 - the red trail from Wołosate to Bukowska pass, about $1 \mathrm{~km}$ before the pass, $900 \mathrm{~m}$, in the brush $P$. sp. $(<10)$, vid. J. Soboń \& B. Gierczyk, 19.07.2006, 08.07.2008 (Gierczyk \& Soboń 2008); Przełęcz Bukowska pass, by the Włosatka stream, by a bridge, on P. hybridus, leg. R. Piwowarczyk, 14.08.2009. 